\title{
Erasing the glassy state in magnetic fine particles
}

\author{
Montserrat García del Muro, Xavier Batlle, and Amílcar Labarta* \\ Departament de Física Fonamental, Facultat de Física, Universitat de Barcelona, Diagonal 647, 08028 Barcelona, Spain
}

(Received 19 January 1999)

\begin{abstract}
$\mathrm{BaFe}_{10.4} \mathrm{Co}_{0.8} \mathrm{Ti}_{0.8} \mathrm{O}_{19}$ magnetic fine particles exhibit most of the features attributed to glassy behavior, e.g., irreversibility in the hysteresis loops and in the zero-field-cooling and field-cooling curves extends up to very high fields, and aging and magnetic training phenomena occur. However, the multivalley energy structure of the glassy state can be strongly modified by a field-cooling process at a moderate field. Slow relaxation experiments demonstrate that the intrinsic energy barriers of the individual particles dominate the behavior of the system at high cooling fields, while the energy states corresponding to collective glassy behavior play the dominant role at low cooling fields. [S0163-1829(99)05421-1]
\end{abstract}

Fine magnetic particle systems show most of the features of glassy systems due to the random distribution of anisotropy axis, interparticle interactions, and surface effects. These main features ${ }^{1}$ include the flattening of the field cooling susceptibility, ${ }^{2}$ an increase in the magnetic viscosity, ${ }^{3}$ the occurrence of aging effects, ${ }^{4}$ the critical slowing down observed by ac susceptibility, ${ }^{5}$ and the increase in the nonlinear susceptibility as the blocking temperature is approached from above. ${ }^{3}$ These features do not seem to be associated with a true spin-glass transition. Nevertheless, some authors claim that they reveal the existence of some kind of collective state. ${ }^{3,6}$ Although this state is mostly attributed to the frustration induced by magnetic interactions between randomly distributed particles, ${ }^{6}$ some studies suggest the dominant role of surface spin disorder. ${ }^{7}$ One of the facts that makes the behavior of these systems complex is the coexistence of the freezing associated with frustration and the intrinsic blocking of the particles. Consequently, depending on the time window of the experimental technique, one or both phenomena are observed. For example, blocking effects usually determine the results of Mössbauer spectroscopy, since the measured blocking temperature decreases with increasing interactions, ${ }^{8}$ while freezing phenomena determine the thermal dependence of the cusp of the real part of the ac susceptibility for concentrated samples, which moves to higher temperatures with increasing interactions. ${ }^{9}$

In this paper, we show that the glassy state of strong interacting particles can be destroyed by a field-cooling process at a moderate magnetic field, which precludes a true phase transition. We also demonstrate that the dynamics of these systems is strongly affected by the initial magnetic moment configuration, in such a way that the glassy state determines the dynamic behavior only in low-cooling-field experiments, while at high cooling fields the dynamics is mostly dominated by the intrinsic energy barriers of the individual particles. These conclusions result from comparing the effective distribution of energy barriers obtained from the $T \ln \left(t / \tau_{0}\right)$ analysis of the magnetic relaxation ${ }^{10}$ measured after field cooling the sample at different fields. The results of some aging experiments also reinforce these conclusions.

The sample of $\mathrm{BaFe}_{10.4} \mathrm{Co}_{0.8} \mathrm{Ti}_{0.8} \mathrm{O}_{19}$ particles used in this work was prepared by the glass crystallization method. ${ }^{11}$ This technique has proved to be an excellent method of ob- taining $M$-type barium ferrite nanoparticles with plateletlike shape and a narrow size distribution. ${ }^{12,13}$ The particle size distribution as determined from transmission electron microscopy (TEM) and x-ray diffraction was log normal with a mean platelet diameter of $10.2 \mathrm{~nm}$ (mean volume of 105 $\left.\mathrm{nm}^{3}\right), \sigma=0.48$, and an aspect ratio of 4 . In order to maximize interparticle interactions the sample was studied in powder form in which large agglomerates were observed by TEM. $^{12}$ Particle stacks, clusters, and quasispherical conglomerates were observed, as expected for the aspect ratio of this sample. ${ }^{14}$ The powder was mixed with a glue in order to avoid particle rotation toward the applied field axis during magnetic measurements. Magnetization measurements were carried out with a superconducting quantum interference device (SQUID) magnetometer under magnetic fields up to 70 kOe. High-field magnetization measurements (0-240 kOe) were made using a water-cooled Bitter magnet with a vibrating sample magnetometer. Thermal dependence of the magnetization was recorded after zero-field cooling (ZFC) and field cooling (FC) the sample at $200 \mathrm{Oe}$ and $70 \mathrm{kOe}$. Isothermal hysteresis loops were measured at several temperatures, after ZFC the sample from room temperature and after a FC process at $70 \mathrm{kOe}$. The time dependence of the thermoremanence in the temperature range $5-230 \mathrm{~K}$ was measured by FC the sample at different fields $\left(200,500,10^{4}\right.$, and 5 $\left.\times 10^{4} \mathrm{Oe}\right)$ from room temperature down to the measuring temperature and then switching off the field. Aging phenomena were studied at $150 \mathrm{~K}$ for different waiting times after a $\mathrm{FC}$ process at $200 \mathrm{Oe}$ and $10 \mathrm{kOe}$.

We show in Fig. 1 the hysteresis loop at $5 \mathrm{~K}$ with a maximum applied field of $200 \mathrm{kOe}$, displaying the typical features of fine particle systems: the saturation magnetization is about half of the bulk value, the high-field differential susceptibility is about the double, and the coercive field is about 4 times larger. Furthermore, the onset of irreversibility occurs at $125 \mathrm{kOe}$, which is much larger than the typical values for bulk ferrites (thousands of Oe), and the hysteresis loop recorded after FC the sample at $70 \mathrm{kOe}$ is shifted ca. $500 \mathrm{Oe}$ in the opposite direction to the cooling field (magnetic training effect). The two latter facts have recently been attributed to the presence of a surface spin-glass layer, ${ }^{7}$ although a glassy state arising from interparticle magnetic frustration may also explain this anomalous behavior. The 


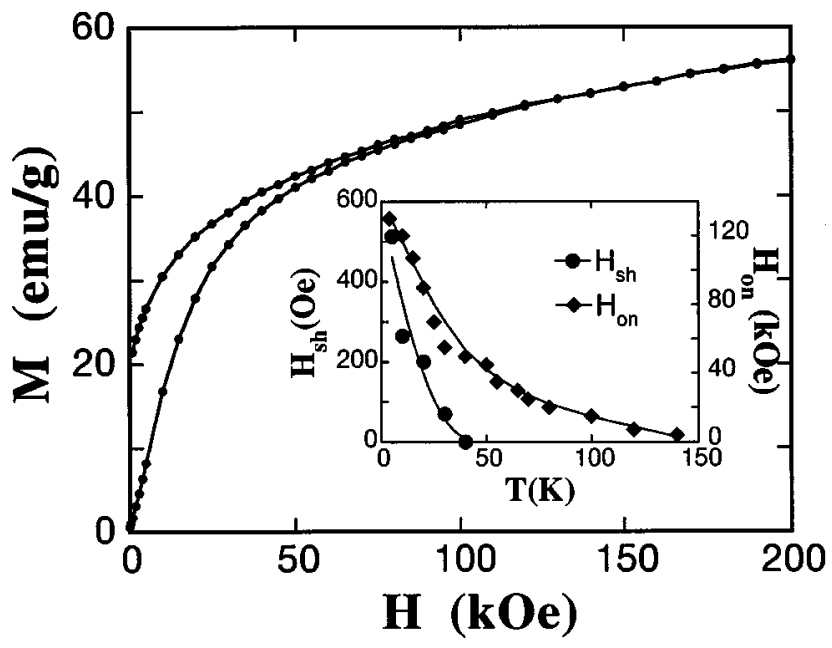

FIG. 1. Magnetization vs magnetic field at $5 \mathrm{~K}$. The inset shows the thermal dependence of the onset of the irreversibility $\left(H_{o n}\right)$ and the shifting of the hysteresis loops after a FC process at $70 \mathrm{kOe}$ $\left(H_{s h}\right)$.

thermal dependence of the onset of the irreversibility and the shifting of the hysteresis loops are shown in the inset of Fig. 1. The latter rapidly decays to zero as the glassy behavior disappears with temperature, while the former evolves more slowly toward the intrinsic anisotropy field.

The low-field susceptibility (200 Oe) displayed a wide maximum in the ZFC curve at ca. $205 \mathrm{~K}$ and a flattened FC curve below this temperature, ${ }^{15}$ being $T_{0}=-170 \pm 30 \mathrm{~K}$, the value of the Curie temperature extrapolated from the reciprocal susceptibility, ${ }^{15}$ which indicates strong interparticle interactions. Taking into account the aggregation state of the powder samples, direct exchange through the surface of neighboring particles and dipolar interactions are present. When susceptibility is measured at $70 \mathrm{kOe}$ the ZFC-FC curves are still irreversible and the ZFC curve displays a maximum at ca. $40 \mathrm{~K}$ (see Fig. 2). These results also support the occurrence of a glassy state in this assembly of nanoparticles.

In order to obtain further insight into the nature of this glassy state, the time dependence of the thermoremanence

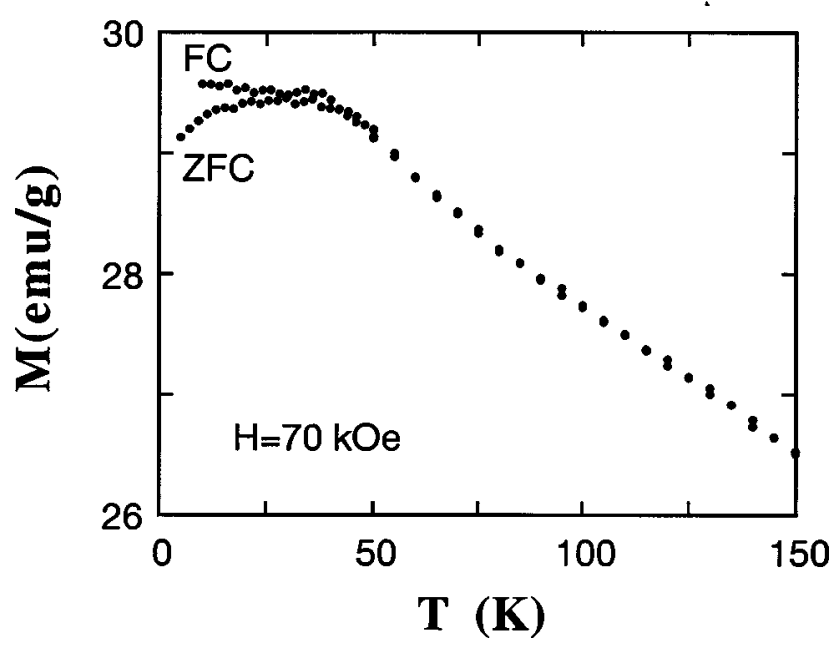

FIG. 2. ZFC-FC magnetization curves measured at $70 \mathrm{kOe}$ as a function of temperature.

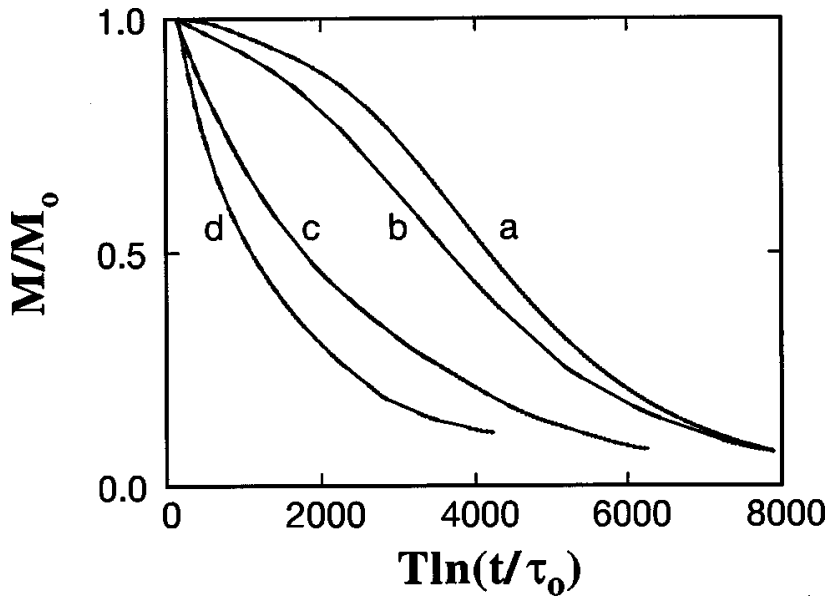

FIG. 3. $T \ln \left(t / \tau_{0}\right)$ scaling of the magnetic relaxation data measured in the temperature range $5-230 \mathrm{~K}$ after FC the sample at 200 Oe (a), $500 \mathrm{Oe}(\mathrm{b}), 10 \mathrm{kOe}(\mathrm{c})$, and $50 \mathrm{kOe}(\mathrm{d}) . M_{0}$ is an arbitrary normalization factor.

was analyzed in terms of the $T \ln \left(t / \tau_{0}\right)$ scaling, ${ }^{10}$ since this procedure allows calculation of the effective distribution of energy barriers. ${ }^{18}$ Among the variety of methods to study the complex hierarchy of energy minima in glassy systems, the $T \ln \left(t / \tau_{0}\right)$ scaling leads to a time-independent effective distribution of energy barriers which averages the contributions due to volume, shape, and surface anisotropy and interparticle interactions. Through this simple method, it is possible to ascertain the differences between those magnetic systems that are labeled as glassy, for which the existence of a large number of quasidegenerated states makes the dynamics complex since the actual energy barrier distribution is time dependent. The scaling procedure consists in choosing the value of $\tau_{0}$ (characteristic attempt time) that makes all the experimental relaxation curves, obtained at different temperatures, scale onto a single master curve, which stands for the whole relaxation curve at the lowest measuring temperature. The effective distribution of energy barriers is then obtained by calculating the derivative of the experimental master curve with respect to $T \ln \left(t / \tau_{0}\right)$.

Figure 3 shows the results of the scaling of the relaxation data measured after FC the sample at different fields. The value of $\tau_{0}$ used in this scaling is $10^{-12} \mathrm{~s}$, which is consistent with those values deduced from the frequency dependence of the maximum of the real part of the ac susceptibility (Volger-Folcher law) and the activated dynamic scaling of these data. ${ }^{16}$ From this figure, it is evident that the cooling field drastically modifies the relaxation curves, which demonstrates that, when interparticle interactions are strong, the initial arrangement of the particle moments (FC initial state) determines the time evolution of the magnetization, in contrast with the noninteracting case for which the observed results are independent of the cooling field. ${ }^{17}$ The effective distributions of energy barriers, $f(E)$, obtained from the master curves are shown in Fig. 4. At low cooling fields, $f(E)$ extends to extremely high energies, and the energy of the maximum is much higher (one order of magnitude) than that expected from bulk anisotropy, $K f(v)$, where $K$ is the bulk anisotropy constant ${ }^{19}$ and $f(v)$ is the volume distribution derived from TEM. ${ }^{12}$ In contrast, the resulting $f(E)$ pro- 


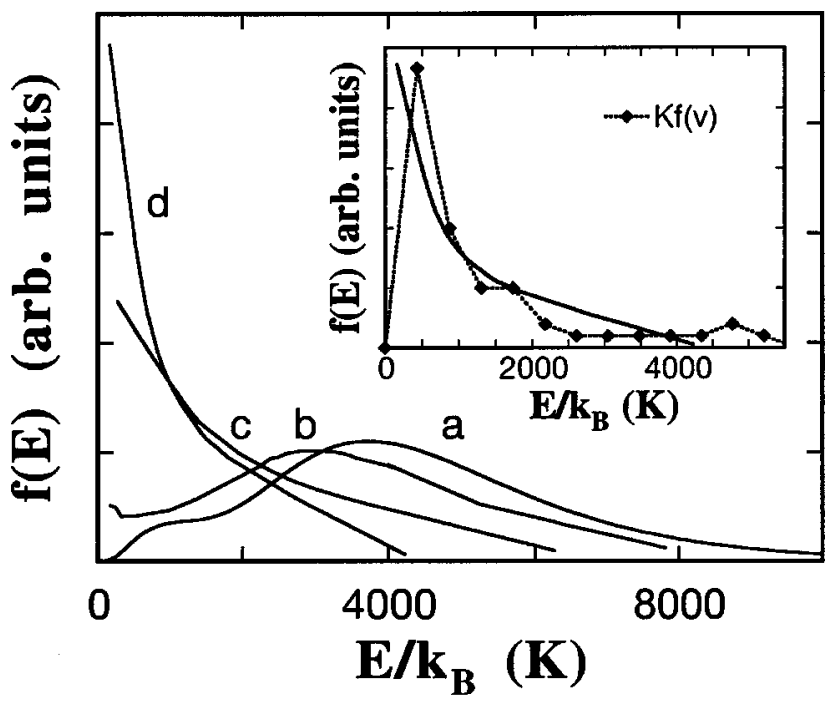

FIG. 4. Efective distribution of energy barriers obtained by numerical derivative of the scaling curves shown in Fig. 3. The labels of the curves are as in Fig. 3. In the inset, the energy distribution obtained after FC the sample at $50 \mathrm{kOe}$ [curve (d)] is compared to $K f(v)$ (dots conected by a dashed line).

gressively resembles $K f(v)$ as the cooling field increases (see inset of Fig. 4). The component of the energy barrier distribution centered at high energies, which is dominant at low cooling fields, is then attributed to the collective behavior associated with the glassy state due to the fact that particle magnetization is mostly randomly distributed in the field-cooled state. Nevertheless, the energy barrier distribution observed at high cooling fields, which is centered at much lower energies, corresponds to the intrinsic anisotropy of the individual particles. In the high-field-cooled state, the magnetization is mostly parallel aligned. Therefore, the overall dipolar interactions are demagnetizing and their effect can be considered through a mean magnetic field which reduces the height of the energy barrier associated to the intrinsic anisotropy of the particles. As a consequence, a slight shift to lower energies is observed in the energy barrier distribution ${ }^{20}$ (see inset of Fig. 4). At intermediate fields, a bimodal $f(E)$ which arises from both contributions is observed, their relative importance being determined by the strength of the cooling field [see $f(E)$ curves corresponding to 200 and 500 Oe in Fig. 4].

Aging is commonly considered a characteristic feature of systems with enough frustration as to induce a multivalley energy structure at low temperature. In particular, it has recently been observed in strong interacting particle systems. ${ }^{4}$ The system studied in this work also shows aging below about the temperature of the maximum of the ZFC curve, which is proof of the existence of a glassy state. Figure 5 shows the relaxation curves at $150 \mathrm{~K}$ after a FC process at 200 Oe for waiting times of $5 \times 10^{2}$ and $10^{3} \mathrm{~s}$. The characteristic trends of aging are present in these curves: (i) the relaxation rate decreases as the waiting time increases and (ii) the relaxation curves plotted in a logarithmic time scale show an inflection point at about the waiting time. However, when the same experiments are repeated with a cooling field of $10 \mathrm{kOe}$, no significant differences in the relaxation curves are observed as the waiting time is increased

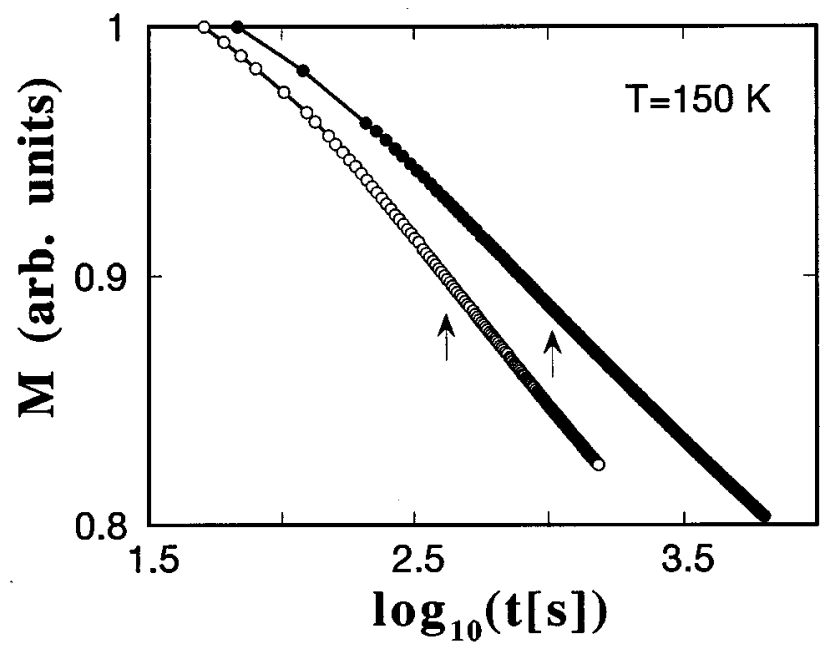

FIG. 5. Relaxation curves at $150 \mathrm{~K}$ after a FC process at $200 \mathrm{Oe}$ for waiting times of $5 \times 10^{2} \mathrm{~s}$ (open circles) and $10^{3} \mathrm{~s}$ (solid circles). Arrows indicate the inflection point of the curves, which is located at about the waiting time.

(see Fig. 6). Therefore, the characteristic aging associated with the glassy state only occurs at low enough cooling fields.

In conclusion, cooling fields monitor the dynamics of interacting fine magnetic particles through determining the initial state of the magnetic moment arrangement. Consequently, at high cooling fields the dynamics of the system is mostly dominated by the intrinsic energy barriers of the individual particles, while at low cooling fields, the energy states corresponding to collective glassy behavior play the dominant role. Care should thus be taken when comparing relaxation data from isothermal remanent magnetization and thermoremanent magnetization, since the initial magnetic state may be very different depending on the field strength. These systems are relevant because, although they display an important degree of magnetic frustration, the collective state may be destroyed by the application of a moderate field, which precludes a true spin glass behavior.

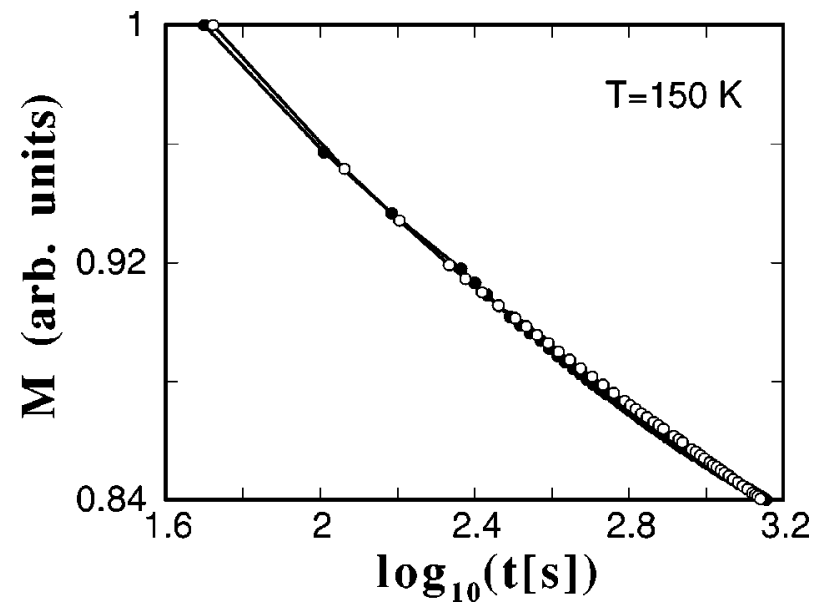

FIG. 6. Relaxation curves at $150 \mathrm{~K}$ after a FC process at $10 \mathrm{kOe}$ for waiting times of $5 \times 10^{2} \mathrm{~s}$ (open circles) and $10^{3} \mathrm{~s}$ (solid circles). 
We would like to thank Professor P. Görnert from I.P.H.T.-Jena (Germany) for making the samples used in this work available to us. We are indebted to Dr. H. Van Tol from the Grenoble High Field Laboratory (C.N.R.S.- Max
Planck Institut) for his help during the high-magnetic-field measurements. Financial support of both the Spanish CICYT through the MAT97-0404 project and the Catalonian CIRIT through the SGR119 projects is recognized.
*Author to whom correspondence should be addressed. Electronic address: amilcar@ffn.ub.es

${ }^{1}$ For general references see J.L. Dormann, D. Fiorani, and E. Tronc, Adv. Chem. Phys. 98, 283 (1997); in Magnetic Properties of Fine Particles, edited by J.L. Dormann and D. Fiorani (North-Holland, Amsterdam, 1992).

${ }^{2}$ P. Prené, E. Tronc, J.P. Jolivet, J. Livage, R. Cherkaoui, M. Noguès, J.L. Dormann, and D. Fiorani, IEEE Trans. Magn. 29, 2658 (1993).

${ }^{3}$ J.L. Dormann, R. Cherkaoui, L. Spinu, M. Noguès, F. Lucari, F. D'Orazio, D. Fioarani, A. García, E. Tronc, and J.P. Jolivert, J. Magn. Magn. Mater. 187, L139 (1998).

${ }^{4}$ T. Jonsson, J. Mattsson, C. Djurberg, F.A. Khan, P. Nordblad, and P. Svedlindh, Phys. Rev. Lett. 75, 4138 (1995).

${ }^{5}$ C. Djurberg, P. Svedlindh, P. Nordblad, M. F. Hansen, F. Bødker, and S. Mørup, Phys. Rev. Lett. 79, 5154 (1997).

${ }^{6}$ H. Mamiya, J. Nakatani, and T. Furubayashi, Phys. Rev. Lett. 80, 177 (1998); S. Mørup, Europhys. Lett. 28, 671 (1994); S. Mørup, F. Bødker, P.V. Hendriksen, and S. Linderoth, Phys. Rev. B 52, 287 (1995).

${ }^{7}$ F.T. Parker, M.W. Foster, D.T. Margulies, and A.E. Berkowitz, Phys. Rev. B 47, 7885 (1993); R.H. Kodama, A.E. Berkowitz, E.J. McNiff, Jr., and S. Foner, Phys. Rev. Lett. 77, 394 (1996); J.M.D. Coey, ibid. 27, 1140 (1971); Q.A. Pankhurst and R.J. Pollard, ibid. 67, 248 (1991); M. Respaud, J. M. Broto, H. Rakoto, A. R. Fert, L. Thomas, B. Barbara, M. Verelst, E. Snoeck, P. Lecante, A. Mosset, J. Osuna, T. Ould Ely, C. Amiens, and B. Chaudret, Phys. Rev. B 57, 2925 (1998); B. Martínez, X. Obradors, Ll. Balcells, A. Rouanet, and C. Monty, Phys. Rev. Lett. 80, 181 (1998).
${ }^{8}$ S. Mørup and E. Tronc, Phys. Rev. Lett. 72, 3278 (1994).

${ }^{9}$ T. Jonsson, P. Nordblad, and P. Svedlindh, Phys. Rev. B 57, 497 (1998); J.L. Dormann, L. Bessais, and D. Fiorani, J. Phys. C 21, 2015 (1988); J.L. Dormann, L. Spinu, E. Tronc, J.P. Jolivet, F. Lucari, F. D’Orazio, and D. Fiorani, J. Magn. Magn. Mater. 183, L255 (1998).

${ }^{10}$ A. Labarta, O. Iglesias, Ll. Balcells, and F. Badia, Phys. Rev. B 48, 10240 (1993). See, for spin glasses, J.J. Préjean and J. Souletie, J. Phys. (France) 41, 1335 (1980); R. Omari, J.J. Préjean, and J. Souletie, ibid. 45, 1809 (1984).

${ }^{11}$ B.T. Shirk and W.R. Buessen, J. Am. Ceram. Soc. 53, 192 (1970); P. Görnert, E. Sinn, and M. Rösler, Key Eng. Mater. 58, 129 (1991).

${ }^{12}$ P. Görnert, H. Pfeiffer, E. Sinn, R. Müller, W. Shüppel, M. Rösler, X. Batlle, M. García del Muro, J. Tejada, and S. Galí, IEEE Trans. Magn. MAG-30, 714 (1994).

${ }^{13}$ X. Batlle, M. García del Muro, J. Tejada, H. Pfeiffer, P. Görnert, and E. Sinn, J. Appl. Phys. 74, 3333 (1993).

${ }^{14}$ T. Ido, O. Kubo, and H. Yokoyama, IEEE Trans. Magn. MAG22, 704 (1986).

${ }^{15}$ X. Batlle, M. García del Muro, and A. Labarta, Phys. Rev. B 55, 6440 (1997).

${ }^{16}$ X. Batlle, M. García del Muro, A. Labarta, and P. Görnert, J. Magn. Magn. Mater. 157/158, 191 (1996).

${ }^{17}$ H. Mamiya and I. Nakatami, J. Appl. Phys. 81, 4733 (1997).

${ }^{18}$ O. Iglesias, F. Badia, A. Labarta, and Ll. Balcells, Z. Phys. B 100, 173 (1996).

${ }^{19}$ X. Batlle, X. Obradors, J. Rodríguez-Carvajal, M. Pernet, M.V. Cabañas, and M. Vallet, J. Appl. Phys. 70, 1614 (1991).

${ }^{20}$ R. Ribas and A. Labarta, J. Appl. Phys. 80, 5192 (1996). 